\title{
Exposición ocupacional a polvo de madera y cáncer de senos paranasales
}

\section{Occupational exposure to wood dust and Paranasal Sinus Cancer}

\author{
Yosimar Rojas-García ${ }^{1-3}$, Andreína Peñalver-Paolini ${ }^{2-3}$ \\ 1. Hospital Universitario Puerta de Hierro. Madrid. España \\ 2. Hospital Universitario de Fuenlabrada. Madrid. España \\ 3. Unidad Docente de Medicina del Trabajo de la Comunidad de Madrid. Madrid. España \\ Recibido: 02-03-15 \\ Aceptado: 06-03-15
}

\section{Correspondencia}

Yosimar Rojas García

Hospital Universitario Puerta de Hierro. Madrid. España

Correo electrónico: yosi.med@hotmail.com

Este trabajo se ha desarrollado dentro del Programa Científico de la Escuela Nacional de Medicina del Trabajo del Instituto de Salud Carlos III en convenio con Unidad Docente de Medicina del Trabajo de la Comunidad de Madrid.

Resumen

Introducción: Los tumores malignos de nariz y senos paranasales son relativamente poco frecuentes, representando el $0,2 \%-0,8 \%$ de los tumores malignos del organismo y el $3 \%$ de los tumores del tracto respiratorio superior. La exposición ocupacional a determinadas sustancias, entre las que se incluye la exposición a polvo de madera, puede explicar hasta un $40 \%$ de los casos.

Objetivo: Revisar la literatura científica reciente de trabajos que aborden la exposición laboral a polvo de madera y su posible relación con el desarrollo de cáncer de senos paranasales.

Materiales y métodos: Búsqueda bibliográfica de artículos publicados entre 2009-2014 realizada en las principales bases de datos biomédicas (Medline, Lilacs, Ibecs, Scielo, OSH-Update, y Cochrane library) y selección en base a criterios de inclusión y exclusión previamente establecidos.

Resultados: Se obtiene un total de 7 artículos, 2 de casos y controles, 3 longitudinales retrospectivos, 1 serie de casos y 1 estudio transversal.

Conclusiones: Existe asociación estadísticamente significativa entre la exposición laboral a polvo de madera y el cáncer de senos paranasales, susceptibilidad que se incrementa con mayor tiempo de exposición. Alteraciones genéticas derivadas de esta exposición como la mutación del gen TP53 y KRAS, inmupositividad de P53, y polimorfismo de enzimas del metabolismo CYP1A se encuentran también implicadas en el desarrollo de esta patología.

Med Segur Trab (Internet) 2015; 61 (238) 112-124

Palabras claves: Exposición ocupacional, polvo de madera, Neoplasias de los Senos Paranasales.

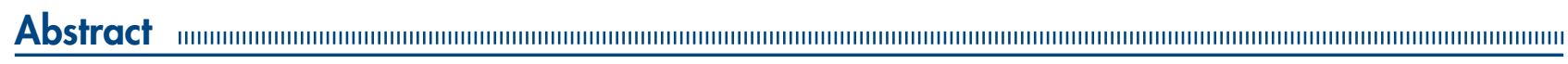

Introduction: Nasal Cavity and Paranasal Sinus Cancer are relatively uncommon. They account for just $0.2 \%-0.8 \%$ of all cancers and for 3\% of the upper respiratory tract tumours. Occupational exposure to certain substances, such as the exposure to wood dust, may account for $40 \%$ of cases. 
Objective: Review the recent scientific literature dealing with occupational exposure to wood dust and its relation with the development of paranasal sinus cancer.

Material and Methods: Bibliographic search of articles published between 2009-2014 in the main biomedical databases (Medline, Lilacs, Ibecs, Scielo, OSH-Update, and Cochrane library) and selected articles according with inclusion-and-exclusion criteria.

Results: 7 articles obtained 2 cases-control articles, 3 longitudinal retrospective articles, 1 case series studies and 1 transversal study.

Conclusions: There is a relevant statistical association between wood dust labour exposure and paranasal sinus cancer. Such susceptibility may increase with heavier exposure or longer exposure time. TP53 and K-ras gene mutation, p53 inmunopositivity and polymorphism enzymes of CYP1A metabolism are genetic alterations involved with the exposure which are as well part of the pathology development.

Med Segur Trab (Internet) 2015; 61 (238) 112-124

Key words: Occupational exposure, wood dust, Paranasal Sinus Neoplasms. 


\section{INTRODUCCIÓN}

Los senos paranasales son cavidades aéreas ubicadas dentro de los huesos del cráneo y la cara. Existen cuatro pares de senos paranasales, que reciben su nombre de acuerdo al hueso que los rodean: maxilares, frontales, esfenoidales y etmoidales.

Los tumores malignos de nariz y cavidades paranasales o nasosinusales son relativamente poco frecuentes, representando entre el $0,2 \%$ y $0,8 \%$ de los tumores malignos del organismo y, aproximadamente, el 3\% de los tumores del tracto respiratorio superior. Son más frecuentes en hombres que en mujeres en proporción de 2:1; se presentan habitualmente en edades medias de la vida (cuarta década) ${ }^{1}$ y entre los factores predisponentes, es importante la exposición ocupacional a determinadas sustancias, encontrándose dicho antecedente hasta en el $40 \%$ de los casos. ${ }^{2}$ En cuanto a la localización, $40 \%$ de los de origen ocupacional se desarrollan en el seno etmoidal; en cambio de $20 \%$ a $50 \%$ de los esporádicos, surgen en el seno maxilar. ${ }^{3}$ El polvo de madera dura se ha vinculado a una mayor incidencia de adenocarcinoma de etmoides, mientras que el polvo de madera blanda a carcinomas escamosos.

La industria de la madera engloba múltiples procesos, que van desde la tala de árboles, hasta la producción de muebles y materiales de construcción, entre otros. Las fases de transformación de la madera para fabricar productos son variadas y en ellas los trabajadores se pueden encontrar expuestos a diferentes sustancias, principalmente a polvo de madera.

El polvo de madera, constituido fundamentalmente por celulosa, poliosa y lignina, puede contener gran variedad de sustancias químicas que pueden ser nocivas, algunas propias de la madera como las resinas y alcaloides y otras aplicadas por el hombre para protegerla, como antifúngicos e insecticidas, o bien sustancias propias de la actividad industrial como barnices, colorantes artificiales, etc. El polvo de madera dura se ha clasificado como cancerígeno del grupo 1 por la Agencia Internacional para la Investigación sobre el Cáncer (IARC) ${ }^{4}$ puesto que hay suficiente evidencia de que produce cáncer en humanos; en cuanto a parámetros internacionales se han establecido límites por la Conferencia Americana de Higienistas Industriales Gubernamentales (ACGIH) que oscilan entre $1 \mathrm{mg} / \mathrm{m}^{3}$ para maderas duras y $5 \mathrm{mg} / \mathrm{m}^{3}$ para las blandas, mientras en España se encuentra establecido un valor límite de $5 \mathrm{mg} / \mathrm{m}^{3}$ para exposición a polvo de madera dura. ${ }^{5}$

La clasificación de la madera se establece en función de criterios botánicos, considerándose maderas duras las provenientes de especies arbóreas caducifolias como el roble, haya, nogal, cerezo, castaño, mientras que las maderas blandas provienen de especias arbóreas coníferas, entre las que podemos indicar el pino, abeto y el cedro. ${ }^{6} \mathrm{El}$ haya y el roble se consideran carcinógenos humanos confirmados y figuran en la guía de la ACGIH americana con la anotación A1. El resto figuran en la guía de la ACGIH con la anotación A2 (carcinógeno humano sospechoso).

En España existen aproximadamente medio millón de trabajadores expuestos en el ámbito laboral a la inhalación de polvo de madera. Principalmente en los sectores de construcción, industria de fabricación de muebles, carpintería, silvicultura y aserraderos, además de otras actividades que indirectamente emplean a carpinteros, ebanistas u otros trabajadores de la madera. ${ }^{7}$

Respecto a esta situación, en la legislación española vigente, el RD 374/2001, de 6 de abril, regula sobre la protección de la salud y seguridad de los trabajadores contra los riesgos relacionados con los agentes químicos en sus diversas formas de presentación -entre ellas el polvo - presentes en el lugar de trabajo. ${ }^{9}$ Este RD traspuso al ordenamiento jurídico español la Directiva del Consejo 98/24/CE de 7 de abril y la Directiva 2000/39/ CE de la Comisión Europea de 8 de junio.

Respecto a sustancias cancerígenas, como es el caso del polvo de madera dura, es de aplicación el R. D. 665/1997, ${ }^{10}$ de 12 de mayo, sobre la protección de los trabajadores 
contra los riesgos relacionados con la exposición a agentes cancerígenos durante el trabajo y la modificación contenida en el R. D. 349/2003, de 21 de marzo, ${ }^{11}$ que incluye por primera vez un valor límite de exposición profesional para el polvo de madera dura y por el que se amplía su ámbito de aplicación a los agentes mutágenos. La neoplasia maligna de cavidad nasal debido a polvo de madera dura, se incluye en el cuadro de enfermedades profesionales en el sistema de la Seguridad Social en el RD 1299/2006, de 10 de noviembre. ${ }^{12}$

Desde hace décadas se vienen realizado estudios orientados a esclarecer el papel que pueda jugar la exposición a polvo de madera en el desarrollo del cáncer nasosinusal, y algunos autores concluyen que la utilización de maderas duras como el roble y el haya están etiológicamente relacionados con el desarrollo de estos tumores, postulando que dicha exposición ocupacional era el factor de riesgo más importante. ${ }^{14-16}$

El objetivo principal de este estudio es buscar en la literatura científica reciente trabajos que aborden la exposición laboral a polvo de madera y su posible relación con el desarrollo de cáncer de senos paranasales, a objeto de describir la mayor evidencia científica que en el momento actual se tenga sobre esta relación, así como buscar datos relacionados con la dosis-respuesta, tiempo de exposición, tipo histológico más frecuente, posible asociación a mutaciones genéticas y, en la medida de lo posible, definir los procesos de trabajo dentro de la industria maderera que tienen mayor riesgo para desarrollar cáncer nasosinusal.

\section{MATERIALES Y MÉTODOS}

Se realiza una revisión sistemática en el mes de Noviembre del 2014 de la literatura científica publicada durante el periodo comprendido entre 2009-2014 a partir de bases de datos bibliográficas como Medline-PubMed, LILACS, IBECS, OSH-Update y Cochrane library, así como del repositorio SciELO.

Se utilizaron descriptores del Medical Subject Headings (MeSH) y Descriptores en Ciencias de la Salud (DeCS) como principales términos de búsqueda para las bases de datos que utilizan dichos tesauros (Medline-PubMed, LILACS, IBECS y Cochrane library). Los descriptores que consideramos fueron: neoplasms, otorhinolaryngologic neoplasms, wood, wood/adverse effects, wood/ toxicity. (Tabla I).

La colección bibliográfica resultante se completó mediante una búsqueda libre en Google Schoolar y otras fuentes bibliográficas de internet.

Se definieron criterios de inclusión y exclusión para seleccionar los artículos atendiendo a los objetivos del mismo.

\section{Criterios de inclusión}

- Población trabajadora en la industria de la madera.

- Cáncer de senos paranasales.

- Trabajos publicados entre los años 2009 y 2014.

- Trabajos realizados en humanos.

- Artículos en español, inglés, alemán e italiano.

- Se incluyeron para este estudio los siguientes documentos: meta-análisis, ensayo clínico, estudios experimentales, casos y controles, cohortes, estudios observacionales.

\section{Criterios de exclusión}

- Población general.

- Patologías generales del sistema respiratorio. 
- Artículos de opinión, ecológicos, cartas al director, protocolos, comunicaciones, notas técnicas.

- Artículos duplicados.

Se realizó una revisión por pares de forma independiente de cada uno de los artículos para establecer el criterio de pertinencia para su inclusión en el estudio y se aplicaron criterios de consenso en las discrepancias, además se realizó la asignación del nivel de evidencia científica correspondiente a través de los criterios Scottish Intercollegiate Guidelines Network (SIGN). ${ }^{17}$

\section{RESULTADOS}

Se encontró un total de 149 artículos publicados entre 2009-2014, 147 corresponde a los encontrados en las bases de datos y 2 a la búsqueda libre. Tras la aplicación de los criterios de inclusión y exclusión se descartaron 141 artículos que no los cumplían y no se pudo recuperar el texto completo de 1 de ellos, quedando finalmente una colección de 7 artículos que abordaban como tema principal de estudio la relación del cáncer de senos paranasales y la exposición a polvo de madera; con niveles de evidencia entre 2 y 3 .

Tabla I. Estrategias de búsquedas utilizadas para cada una de las bases de datos bibliográficas y artículos encontrados

\begin{tabular}{|c|c|c|}
\hline Bases de datos & Estrategia de búsqueda & Resultados obtenidos \\
\hline $\begin{array}{l}\text { MEDLINE } \\
\text { (Vía PUBMED) }\end{array}$ & $\begin{array}{l}\text { ((«neoplasms»[MeSH Terms] OR } \\
\text { "otorhinolaryngologic neoplasms»[MeSH } \\
\text { Terms])) AND (("Wood»[MeSH Terms] OR } \\
\text { "Wood/adverse effects»[MeSH Terms] OR } \\
\text { "Wood/toxicity»[MeSH Terms])) } \\
\text { Filters: published in the last } 5 \text { years; Humans }\end{array}$ & $\begin{array}{l}\text { Sin aplicar filtros: } 409 \\
\text { Tras aplicar filtros } \\
40\end{array}$ \\
\hline LILACS & $\begin{array}{l}\text { Cancer AND Madera OR Madera/efectos } \\
\text { (Palabras) }\end{array}$ & 16 \\
\hline IBECS & Cancer AND Madera & 0 \\
\hline SCIELO & Cancer AND Madera & 0 \\
\hline OSH-UPDATE & $\begin{array}{l}\text { Neoplasms OR otorhinolaryngologic } \\
\text { neoplasms AND wood OR wood effects } \\
\text { adverse OR wood toxicity }\end{array}$ & $\begin{array}{l}91 \\
\text { Tras aplicar filtro 2009-2014: } \\
0\end{array}$ \\
\hline COCHRANE LIBRARY & Cancer AND Madera & 0 \\
\hline $\begin{array}{l}\text { BÚSQUEDAS BIBLIOGRÁFICAS } \\
\text { SECUNDARIAS }\end{array}$ & & 2 \\
\hline
\end{tabular}

De los 7 artículos incluidos en el estudio, 2 son casos y controles, 3 son longitudinales retrospectivos, 1 es una serie de casos y 1 es un estudio transversal. El análisis de los resultados de los diferentes estudios se exponen a continuación de forma cronológica.

Holmila R y cols. (2009). ${ }^{18}$ En una serie de casos analizaron las mutaciones del gen TP53 en 358 casos de cáncer nasosinusal con o sin exposición ocupacional a polvo de madera mediante electroforesis capilar, análisis de polimorfismo de conformación de cadena sencilla y secuenciación directa. La muestra de los tejidos se obtuvieron de 3 países; Dinamarca para el año 1991- 2001 (170 casos), Finlandia en 1989- 2002 (109 casos) y en Francia en los años 1990- 2002 (79 casos).

Los datos sobre la exposición a polvo de madera estaban disponible para $79 \%$ de los casos. El estudio muestra una asociación significativa entre esta exposición y la histología de adenocarcinoma (OR 12.6, IC95\%, 5.0-31.6), el cual se asoció a polvo de madera dura (IC95\% 19-20) mientras que los expuestos a polvo de madera blanda presentaron carcinoma de células escamosas (IC95\% 23-43; p`0,001). 
La mutación TP53 fue más común en el adenocarcinoma (OR 2.0; IC95\%, 1.01-3.07). El riesgo de mutación TP53 se incrementó en asociación con la duración de la exposición a polvo de madera (mayor o igual 24 años, OR 5,1; IC95\%, 1.5-17.1), nivel medio ('2 mg/ $\mathrm{m}^{3}$; OR 3.6, IC95\%, 1,2-10,8) y el nivel acumulativo (mayor o igual $30 \mathrm{mg} / \mathrm{m}^{3}$ por años; OR $3.5 ; \mathrm{IC} 95 \%, 1.2-10.7)$.

Estos autores investigaron la mutación del gen KRAS en la muestra de casos de Dinamarca y encontraron que rara vez se altera, y que de las mutaciones ocurridas en su mayoría eran en adenocarcinomas ( 7 casos de 58, 12\%) y de estos 2 eran de tipo intestinal.

D Errico A y cols. (2009). ${ }^{19}$ Estudiaron en una muestra de 113 casos y 336 controles el riesgo de cáncer nasosinusal por tipo histológico y por exposición previa a factores de riesgo ocupacionales sospechosos. Entre 1996 y 2000, los casos incidentes fueron recogidos de hospitales de toda la región italiana de Piamonte por el Registro Regional de Cáncer Nasosinusal.

El estudio mostró que cualquier cáncer epitelial nasosinusal se relacionaba significativamente con la exposición a polvo de madera (OR 11.4), ajustado por edad y sexo; aumentando el riesgo de adenocarcinoma (OR 58.6) y se duplicó con cada 5 años de exposición ( $\left.\mathrm{p}^{`} 0.0001\right)$.

También encontraron relación dosis-respuesta entre el riesgo de adenocarcinoma y años de exposición. La exposición de baja intensidad aumenta significativamente el riesgo (OR 52.4).

Ekburanawat $\mathbf{W}$ y cols. (2010). ${ }^{20}$ Confirman los hallazgos observados en trabajos anteriores a nuestro periodo de estudio, en los que asociaban la presencia de adenocarcinoma nasosinusal a la exposición previa a polvo de madera. El estudio identifica algunos factores de riesgos para cáncer nasofaríngeo en una población tailandesa, ajustado por hábito tabáquico y nivel de educación, en la que encuentran que dicha exposición en el ambiente ocupacional incrementa el riesgo (OR 1.63, IC95\% 1.022.61).

Cantu G y cols. (2010). ${ }^{21}$ Estudiaron el papel que podían jugar los factores individuales al observar que aunque todos los trabajadores habían estado sometidos a los mismos niveles altos de exposición a polvos orgánicos (madera y cuero), solo algunos de ellos desarrollaban un adenocarcinoma de senos paranasales de tipo intestinal (ITAC), apuntando hacia la hipótesis de que el polimorfismo de enzimas del metabolismo en xenobióticos puede jugar un papel importante y contribuir de esta forma a que se presente un alto grado de variabilidad en la susceptibilidad individual en lo que respecta al riesgo de desarrollar este tipo de cáncer.

Realizaron un estudio longitudinal retrospectivo en una muestra de 345 pacientes con tumores malignos nasosinusales intervenidos quirúrgicamente en la unidad cráneomaxilo-facial del Instituto Nacional del Tumor de Milán, Italia; entre 1997 y 2006, interrogados sobre exposición laboral previa a polvos orgánicos, y comparados en el departamento experimental de patología molecular los genotipos de enzimas del metabolismo CYP1A1 y GSTM1 en 30 pacientes con ITAC etmoidal con los de 79 donantes de sangre sanos, para verificar si estos polimorfismos podían identificar sujetos con alto riesgo para desarrollar ITAC. Encontraron una asociación estadísticamente significativa entre el ITAC de etmoides y exposición a polvos orgánicos (madera y cuero) ( $p^{`} 0.0001$ ), así como una sobreexpresión del polimorfismo de CYP1A, en pacientes con ITAC en comparación con controles, asociado frecuentemente a la supresión del genotipo GSTM1. De este modo sugirieron que este polimorfismo puede estar asociado con un alto grado de susceptibilidad a este tumor. El tiempo medio de exposición fue 7,5 años (rango, 4-18).

Breheret $\mathbf{R}$ y cols. (2011). ${ }^{22}$ Realizaron un estudio longitudinal retrospectivo incluyendo 42 sujetos en los que se conocía la ocupación de 41 de ellos y de los cuales $36(85,7 \%)$ tuvieron exposición ocupacional a polvo de madera. Especificaron un tiempo de exposición de 30,4 +/-14 años. El intervalo medio entre el fin de la exposición y el diagnóstico de adenocarcinoma de etmoides fue de 10,9 años. Aunque no encontraron 
relación entre el grado tumoral y tiempo de evolución $(\mathrm{p}=0,73)$ o exposición a polvo de madera $(p=0,19)$, encontraron que todos los tumores fueron ITAC; mientras en un $24 \%$ de los pacientes el subtipo histológico fue inespecífico.

Giedre Smailyte (2012). ${ }^{23}$ En un estudio retrospectivo, evaluaron la incidencia de cáncer en una cohorte de 1518 carpinteros expuestos a polvo de madera blanda en una fábrica de productos de carpintería de Lituania, de los que 1080 eran hombres y 438 mujeres. El seguimiento de la cohorte se realizó desde el 1 de Enero de 1978 hasta el 31 de Diciembre de 2007. Los casos de cáncer en la cohorte fueron identificados en el registro de Cáncer Lituano. Se calcularon Razones de Incidencia Estandarizada por edad y sexo (SIR- proporciones entre lo observado y esperado de cáncer).

En general la incidencia total de cáncer no fue mayor de la esperada tanto para la cohorte de hombres como para al de mujeres (SIR hombres; 0.81, IC95\% 0.69-0.95 y SIR mujeres; 0.88, IC95\% 0.65-1.16). En el grupo de mujeres carpinteras se encontró un incremento de la SIR para el cáncer de nariz y senos paranasales (SIR 13.88, IC95\% 0.3577.31), en la cohorte de hombres no se observó este incremento. Finalmente no pudieron concluir que hubiera evidencia de un mayor riesgo de cánceres respiratorios entre los trabajadores expuestos a polvo de madera blanda.

Pérez-Escudero J y cols. (2012). ${ }^{24}$ Realizan un estudio transversal con una muestra de 95 pacientes con adenocarcinoma primario de cavidad nasal y senos paranasales de tipo intestinal, encontraron una frecuencia de la mutación TP53 en un 41\% (18/44) y de inmunopositividad de p53 en un $72 \%$ de los sujetos (66/92), encontrando una relación con la exposición a polvo de madera estadísticamente significativa. Los 18 pacientes con mutación de TP53 y la mayoría de los casos con tinción positiva para p53 tuvieron exposición profesional a polvo de madera. Alrededor de 41 casos donde ambos datos fueron obtenidos, 14 mostraron tanto la mutación como la sobreexpresión proteica, otros 14 con sobreexpresión de la proteína pero no mutación y 1 caso con mutación pero no sobreexpresión proteica. Demostrando que la inmunohistoquímica no es un predictor perfecto de la presencia o ausencia de la mutación de TP53.

En la Tabla II se presentan de forma esquemática los principales hallazgos de los diferentes artículos incluidos en el estudio. 


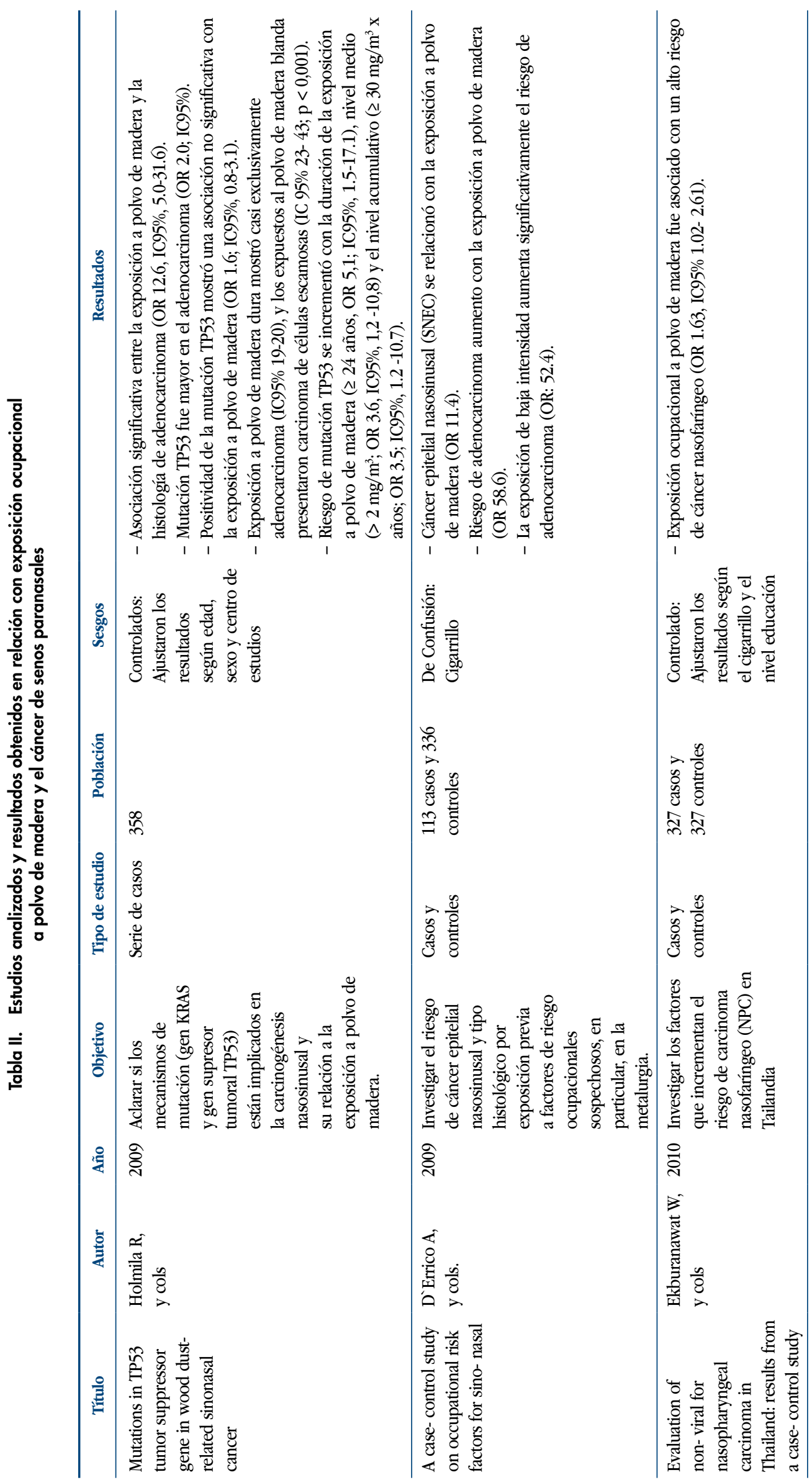




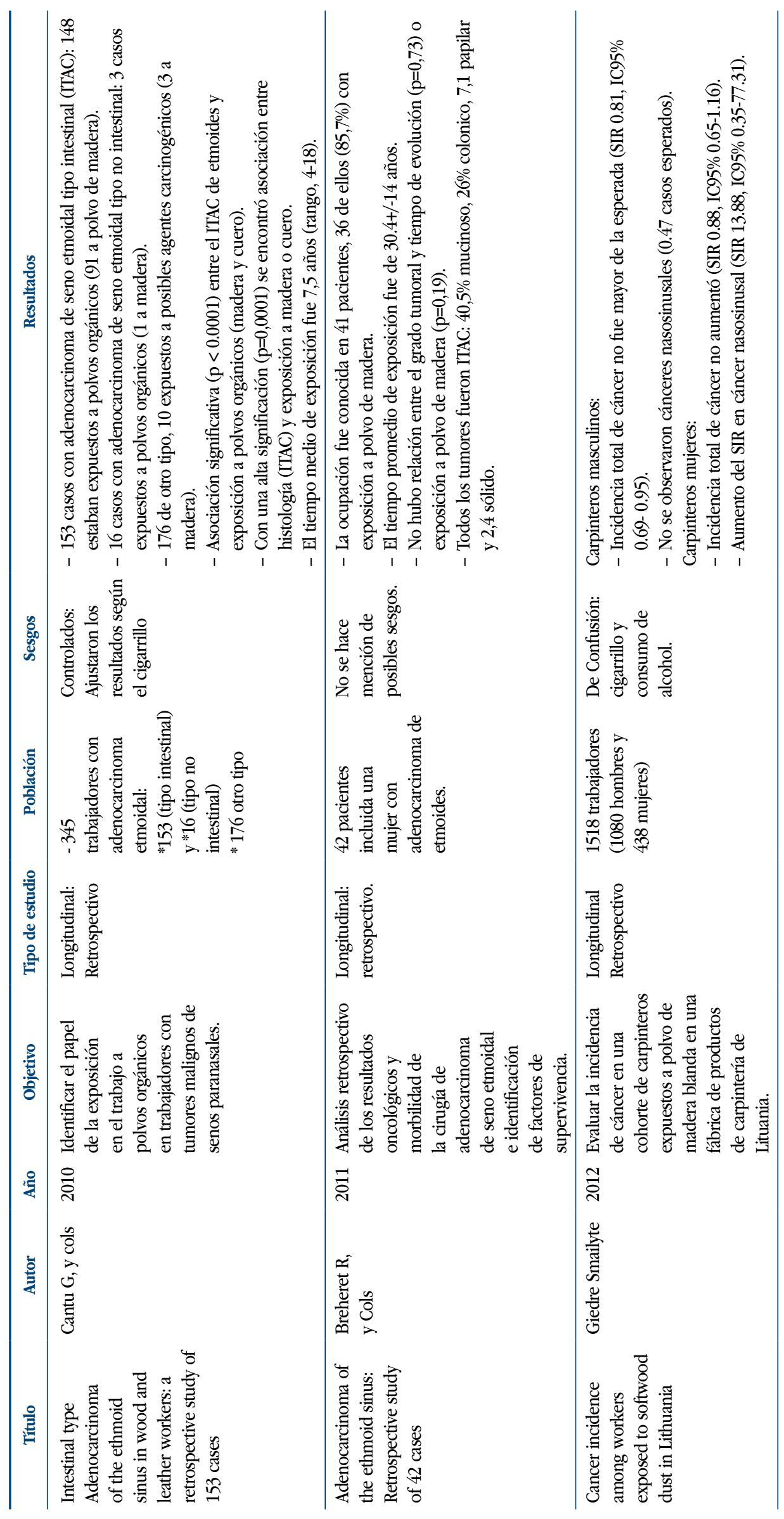




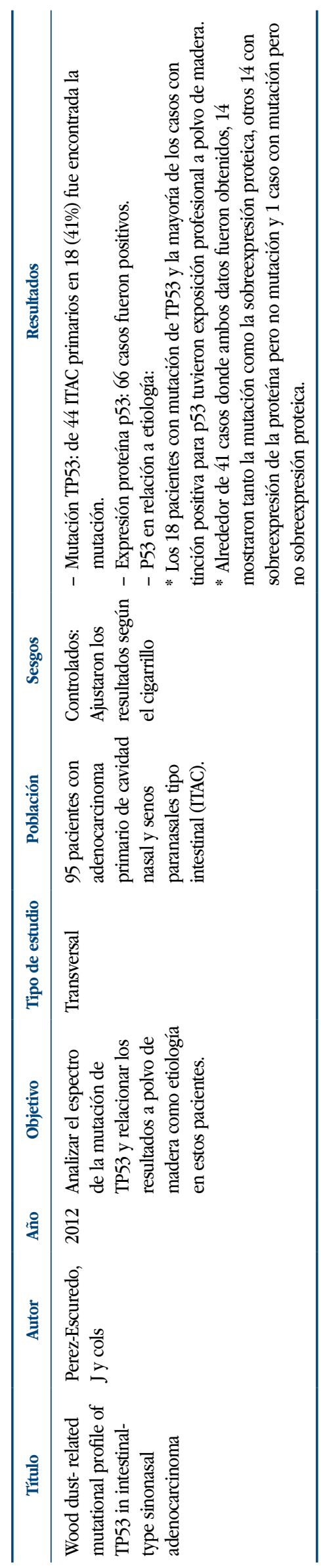




\section{DISCUSIÓN}

Aunque el cáncer de senos paranasales es un tumor raro y muchos de los estudios se realizan sobre muestras pequeñas, la mayor parte de ellos describen una asociación causaefecto estadísticamente significativa entre la exposición laboral a polvo de madera y el cáncer de senos paranasales, riesgo que además parece incrementarse con mayores tiempos de exposición, lo que llevó a la Agencia Internacional para la Investigación sobre el Cáncer (IARC) a clasificar al polvo de la madera como un agente cancerígeno del grupo 1.

El consenso sobre la evidencia de esta relación causa efecto y el que en la actualidad el polvo de la madera se encuentra clasificado dentro del grupo 1, pueden justificar el escaso número de artículos que recientemente se hayan podido publicar abordando el tema y una de las razones por la que tan sólo se encontraron 7 artículos que cumpliesen los criterios de inclusión pertinentes para este estudio.

En general todos los artículos seleccionados confirman la relación entre la exposición a polvo de madera dura y la aparición de cáncer nasosinusal. ${ }^{18-21}$ El único trabajo que aborda de forma específica el estudio de la relación entre el desarrollo de este tipo de tumor con la exposición a polvo de madera blanda, no encuentra relación estadísticamente significativa. $^{23}$

Estudios publicados anteriormente, describen el adenocarcinoma de senos paranasales de tipo intestinal (ITAC) como el tipo de cáncer que más se asocia a la exposición a polvo de madera dura, mientras el tipo histológico epidermoide parece encontrase más relacionado con la exposición a polvo de madera blanda. Estos datos concuerdan con la prevalencia de adenocarcinoma de tipo intestinal nasosinusal descritos por algunos autores de artículos incluidos en este estudio 18,19, 21,22 en relación a la exposición laboral a polvo de madera.

Cantu y su equipo concluyen que el ITAC se origina solamente en el seno etmoidal mientras que los No-ITAC pueden ocurrir en cualquier otro seno paranasal. Incluso que el ITAC de etmoides es el único que tiene una relación claramente relacionada con la exposición laboral a polvo de madera. La mayor limitación de estos estudios radica en que muchos de ellos no especifican la localización exacta del tumor, encontrando sólo dos artículos que describen el adenocarcinoma de etmoides entre los principales objetivos del estudio.

Los trabajos que estudiaban el efecto dosis-respuesta revelan datos que concuerdan con la literatura científica publicada anteriormente. En cuanto al tiempo de exposición a polvo de madera y el riesgo de desarrollar adenocarcinoma de senos paranasales, D`́rrico y Holmila describen una relación directa entre mayor tiempo de exposición y mayor riesgo de padecer este cáncer, que además se incrementa cuando se asocia a una mutación TP53.

Otro aspecto diferencial de los artículos publicados durante el periodo 2009-2014, se centra en el abordaje de aspectos diferentes a los ya conocidos, tales como el papel que puede desempeñar en el proceso causal la presencia de determinados factores individuales y ciertas mutaciones genéticas.

Las alteraciones genéticas principalmente estudiadas se centran en la mutación del gen TP53, inmunopositividad de p53 y el polimorfismo de enzimas del metabolismo CYP1A (supresor del genotipo GSTM1).

Pérez-Escudero J y cols., examinaron tumores primarios ITAC encontrando una asociación con la mutación de TP53 en casi la mitad de la muestra estudiada y todas ellas se producían en pacientes que describían haber tenido una exposición a polvo de madera, por lo que establecen la hipótesis, que radicales libres liberados en condiciones inflamatorias crónicas generadas por dicha exposición laboral, son responsables de la alta frecuencia de la transición de GàA en el TP53 en el ITAC, hipótesis con la que coincide Holmila y cols., que además investigaron la relación del ITAC con mutaciones del gen KRAS, encontrando que rara vez se altera pero que de las mutaciones ocurridas en su mayoría eran en adenocarcinomas ( 7 casos de 58, 12\%) y de estos 2 eran de tipo intestinal (ITAC). 
Dos de los artículos encuentran presencia de inmunopositividad de p53 en la relación de exposición a polvo de madera y la aparición de adenocarcinoma de tipo intestinal, observando que se presentaba con mayor frecuencia en el ITAC subtipo papilar/colónico.

Cantu-Giulio y cols. Compararon el polimorfismo de enzimas del metabolismo CYP1A relacionadas con el supresor del genotipo GSTM1, encontrando una sobreexpresión de este polimorfismo en pacientes con ITAC, por lo que resaltan la importancia de realizar estudios futuros que puedan confirmar el papel de estas enzimas y la susceptibilidad de los sujetos a desarrollar adenocarcinoma nasosinusal, aunque este tipo de estudios puedan suponer un coste relativamente elevado en determinados casos.

En cuanto a la posibilidad de identificar las profesiones que mayor riesgo tienen de desarrollar cáncer de senos paranasales, Ekburanawat $\mathrm{W}$ y su equipo describen una lista de ocupaciones más relacionadas con este tipo de cáncer, destacando los carpinteros, trabajadores de la construcción, ebanistas, aserradores, fabricantes de madera contrachapada y otros trabajos relacionados con la manufactura de la madera, lo que en cierto modo se encuentra avalado por los resultados encontrados en la mayoría de los estudios sobre una evidencia más o menos confirmada de la relación existente entre la exposición a polvo de madera y la aparición de este tipo de tumores. Para Pérez-Escudero, un carpintero varón presenta 500 veces mayor riesgo de padecer cáncer de senos paranasales que el resto de la población masculina y 900 veces más posibilidades en comparación con la población general, no se han encontrado datos objetivos que puedan avalar esta ponderación de riesgo.

En conclusión, el polvo de madera dura es el compuesto más relacionado con la aparición de cáncer de senos paranasales, principalmente adenocarcinoma tipo intestinal (ITAC) y localización etmoidal.

Factores individuales relacionados con mutaciones genéticas en las que podrían estar implicados los genes TP53, inmunopositividad de p53 y el KRAS así como el polimorfismo de enzimas del metabolismo CYP1A relacionado con el supresor del genotipo GSTM1, parece que pueden jugar también un papel en el proceso causal.

Por último, dado que diferentes autores describen un mayor riesgo relacionado con el tiempo de exposición, incluso algunos de ellos señalan un incremento del riesgo en presencia de bajos niveles de exposición, es importante recordar que en ningún caso existen niveles seguros, como lo reconoce el INSHT (instituto nacional de seguridad e higiene en el trabajo) en la guía de Límites de exposición profesional para agentes químicos en España: "los límites de exposición profesional son valores de referencia y no constituyen una barrera definida de separación entre situaciones seguras y peligrosas", donde el único nivel seguro es la no exposición.

\section{REFERENCIAS BIBLIOGRÁFICAS}

1. Sociedad Española de Oncología Médica. Tumores cabeza y cuello- O. R. L. 13 Febrero 2013. http://www. seom.org/es/informacion-sobre-el-cancer/info-tipos-cancer/tumores-orl/orl?start=11\#content Consultada en Enero 2015

2. R Quitral C, M Rahal E, I Morales G, C Daszenies S, MP Vallejos U. Tumores malignos de nariz y cavidades paranasales. Otorrinolaringol. Cir. Cabeza Cuello 2003; 63: 21-28. (http://www.sochiorl.cl/ uploads/63-01_05.pdf)

3. Lazos M. Adenocarcinomas sinonasales. Rev Med Hosp Gen Méx 2011;74(1):35-41.

4. Toscani D. El riesgo de cáncer en los trabajadores de la industria de la madera. 2007; 41: 39. http://www ladep.es/ficheros/documentos/El\%20riesgo\%20de\%20c\%E1ncer\%20en $\% 201$ os\%20trabajadores $\% 20 \mathrm{de} \% 20$ la\%20industria\%20de\%20la\%20madera.pdf

5. Límites de Exposición Profesional para Agentes Químicos adoptados por el Instituto Nacional de Seguridad e Higiene en el Trabajo para el año 2014. http://www.insht.es/portal/site/Insht/menuitem.1f1a3bc79ab34c578c2e8884060961ca/?vgnextoid=2f312741f9f34410VgnVCM1000008130110aRCRD\&vgnextchanne l=75164a7f8a651110VgnVCM100000dc0ca8c0RCRD Consultada en Enero 2015 
6. Guía técnica para la evaluación y prevención de los riesgos relacionados con la exposición durante el trabajo a agentes cancerígenos o mutágenos. http://www.insht.es/InshtWeb/Contenidos/Normativa/ GuiasTecnicas/Ficheros/Agentes_cancerigenos.pdf Consultada en Enero 2015.

7. Centro tecnológico del mármol y la piedra. El polvo de la madera: Riesgo laboral y su prevención. Metal, Construcción y Afines de UGT (MCA-UGT), Federación de Industria. 2010. (http://portal.ugt.org/ saludlaboral/publicaciones/manual_estudio/2010-04.pdf)

8. Ley 31/1995, de 8 de noviembre, de Prevención de Riesgos Laborales. BOE n. ${ }^{\circ} 269$ 10/11/1995. http:// www.insht.es/portal/site/Insht/menuitem.1f1a3bc79ab34c578c2e8884060961ca/?vgnextoid=771be9369a 3d3110VgnVCM100000dc0ca8c0RCRD\&vgnextchannel=1d19bf04b6a03110VgnVCM100000dc0ca8c0RCR D\&tab=tabConsultaCompleta Consultada en Enero 2015

9. Real Decreto $374 / 2001$, de 6 de abril sobre la protección de la salud y seguridad de los trabajadores contra los riesgos relacionados con los agentes químicos durante el trabajo. BOE n. ${ }^{\circ} 104$ 01/05/2001. http:// www.insht.es/portal/site/Insht/menuitem.1f1a3bc79ab34c578c2e8884060961ca/?vgnextoid=feb4f956a51 d5110VgnVCM100000dc0ca8c0RCRD\&vgnextchannel=75164a7f8a651110VgnVCM100000dc0ca8c0RCRD \&tab=tabConsultaCompleta Consultada en Enero 2015.

10. Real Decreto 665/1997, de 12 de mayo, sobre la protección de los trabajadores contra los riesgos relacionados con la exposición a agentes cancerígenos durante el trabajo. http://www.insht.es/portal/site/Insht/ menuitem.1f1a3bc79ab34c578c2e8884060961ca/?vgnextoid=45fab1eae3065110VgnVCM100000dc0ca8c0R CRD\&vgnextchannel=1d19bf04b6a03110VgnVCM100000dc0ca8c0RCRD Consultada en Enero 2015.

11. Real Decreto 349/2003, de 21 de marzo, por el que se modifica el Real Decreto 665/1997, de 12 de mayo, sobre la protección de los trabajadores contra los riesgos relacionados con la exposición a agentes cancerígenos durante el trabajo, y por el que se amplía su ámbito de aplicación a los agentes mutágenos BOE n. ${ }^{\circ} 82$ 05/04/2003. http://www.insht.es/portal/site/Insht/menuitem.1f1a3bc79ab34c578c2e8884060961ca /?vgnextoid=74f5b09a49dc5110VgnVCM100000dc0ca8c0RCRD\&vgnextchannel=1d19bf04b6a03110VgnV CM100000dc0ca8c0RCRD\&tab=tabConsultaCompleta

12. Real Decreto 1299/2006, de 10 de Noviembre, por el que se aprueba el cuadro de enfermedades profesionales en el sistema de la Seguridad Social y se establecen criterios para su notificación y registro. BOE n. 302 19/12/2006. http://www.insht.es/portal/site/Insht/menuitem.1f1a3bc79ab34c578c2e8884060961c a/?vgnextoid=8949e23615dc5110VgnVCM100000dc0ca8c0RCRD\&vgnextchannel=1d19bf04b6a03110Vgn VCM100000dc0ca8c0RCRD\&tab=tabConsultaCompleta Consultada en Enero 2015.

13. Acheson ED, Cowdell RH, Hadfield E, Macbeth RG. Nasal cancer in woodworkers in the furniture industry. Br Med J. 1968 Jun 8;2(5605):587-96.

14. Fontana L, Liétin B, Catilina P, Devif C, Féneon B, Martin F, et al. [Occupational exposure to wood dust and nasal sinus cancer]. Ann Oto-Laryngol Chir Cervico Faciale Bull Société Oto-Laryngol Hôp Paris. 2008 Apr;125(2):65-71.

15. Mensi C, Sieno C, Bordini L, Dicredico N, Pesatori AC, Riboldi L. [Systematic surveillance of occupational cancer: the Lombardy Sinonasal Cancer Registry]. Med Lav. 2010 Feb;101(1):19-25.

16. Barnes L, Choisea SI, Seethala RR. Head and Neck Pathology. Demos Medical Publishing; 2010. 211 p.

17. Scottish Intercollegiate Guidelines Network. SIGN 50: A guideline developer's handbook. http://www. sign.ac.uk. 2008. España.

18. Holmila R, Bornholdt J, Heikkilä P, Suitiala T, Févotte J, Cyr D, et al. Mutations in TP53 tumor suppressor gene in wood dust-related sinonasal cancer. Int J Cancer J Int Cancer. 2010 Aug 1;127(3):578-88.

19. D' Errico A, Pasian S, Baratti A, Zanelli R, Alfonzo S, Gilardi L, et al. A case-control study on occupational risk factors for sino-nasal cancer. Occup Environ Med. 2009 Jul;66(7):448-55.

20. Ekburanawat W, Ekpanyaskul C, Brennan P, Kanka C, Tepsuwan K, Temiyastith S, et al. Evaluation of non-viral risk factors for nasopharyngeal carcinoma in Thailand: results from a case-control study. Asian Pac J Cancer Prev APJCP. 2010;11(4):929-32.

21. Cantu G, Solero CL, Mariani L, Lo Vullo S, Riccio S, Colombo S, et al. Intestinal type adenocarcinoma of the ethmoid sinus in wood and leather workers: a retrospective study of 153 cases. Head Neck. 2011 Apr;33(4):535-42.

22. Breheret R, Laccourreye L, Jeufroy C, Bizon A. Adenocarcinoma of the ethmoid sinus: retrospective study of 42 cases. Eur Ann Otorhinolaryngol Head Neck Dis. 2011 Nov;128(5):211-7.

23. Smailyte G. Cancer incidence among workers exposed to softwood dust in Lithuania. Occup Environ Med. 2012 Jun;69(6):449-51.

24. Pérez-Escuredo J, Martínez JG, Vivanco B, Marcos CÁ, Suárez C, Llorente JL, et al. Wood dust-related mutational profile of TP53 in intestinal-type sinonasal adenocarcinoma. Hum Pathol. 2012 Nov;43(11):1894-901. 